Research Article

\title{
New Exact Solutions of the Fractional Complex Ginzburg-Landau Equation
}

\author{
Chun Huang $\mathbb{D}^{1}$ and Zhao $\mathrm{Li} \mathbb{D}^{2}$ \\ ${ }^{1}$ Faculty of Education, Sichuan Vocational and Technical College, Suining 629000, China \\ ${ }^{2}$ College of Computer Science, Chengdu University, Chengdu 610106, China
}

Correspondence should be addressed to Chun Huang; congnv333@163.com

Received 24 November 2020; Revised 30 December 2020; Accepted 6 January 2021; Published 9 February 2021

Academic Editor: Maria L. Gandarias

Copyright (c) 2021 Chun Huang and Zhao Li. This is an open access article distributed under the Creative Commons Attribution License, which permits unrestricted use, distribution, and reproduction in any medium, provided the original work is properly cited.

\begin{abstract}
In this paper, we apply the complete discrimination system method to establish the exact solutions of the fractional complex Ginzburg-Landau equation in the sense of the conformable fractional derivative. Firstly, by the fractional traveling wave transformation, time-space fractional complex Ginzburg-Landau equation is reduced to an ordinary differential equation. Secondly, some new exact solutions are obtained by the complete discrimination system method of the three-order polynomial; these solutions include solitary wave solutions, rational function solutions, triangle function solutions, and Jacobian elliptic function solutions. Finally, two numerical simulations are imitated to explain the propagation of optical pulses in optic fibers. At the same time, the comparison between the previous results and our results are also given.
\end{abstract}

\section{Introduction}

It is well known that the Ginzburg-Landau equation is one of the most important models to describe optical phenomena [1-7]. In order to better analyze the complex optical phenomena and further study their essence, the best ways are to find the exact traveling solutions [8-15] to the Ginzburg-Landau equation describing the nonlinear optical phenomena. In recent years, a variety of powerful mathematical approaches have been developed to derive the exact solutions to Ginzburg-Landau equation, such as the $\left(G^{\prime} / G^{2}\right)$-expansion method [16], the Modified simple equation method [17], the F-expansion [18], the sineGordon expansion method [19], the extended direct algebraic method [20], and the dynamical system method [21-23].

Consider the following time-space fractional complex Ginzburg-Landau equation [24-28]:

$$
i \frac{\partial^{\delta} u}{\partial t^{2 \delta}}+a \frac{\partial^{2 \delta} u}{\partial x^{2 \delta}}+b F\left(|u|^{2}\right) u=\frac{1}{|u|^{2} u^{*}}\left[\alpha|u|^{2} \frac{\partial^{2 \delta}|u|^{2}}{\partial x^{2 \delta}}-\beta\left(\frac{\partial^{\delta}|u|^{2}}{\partial x^{\delta}}\right)^{2}\right]+\gamma u,
$$

where $0<\delta \leq 1$, describing the order of the fractional derivative, $x$ denotes distance along the fiber, $t$ denotes time in dimensionless form, $a, b, \alpha, \beta$, and $\gamma$ are valued constants, and $F$ is a real-valued algebraic function which must have the smoothness of the function $F\left(|u|^{2}\right) u$ : $\mathbf{C} \longrightarrow \mathbf{C}$. Considering the complex plane $\mathbf{C}$ as a two-dimensional linear space $R^{2}, F\left(|u|^{2}\right) u$ is $k$ times continuously differentiable:

$$
F\left(|u|^{2}\right) u \in \bigcup_{n, m=1}^{\infty} C^{k}\left((-n, n) \times(-m, m) ; R^{2}\right) .
$$

Equation (1) is one of the very many models that govern pulse propagation dynamics through optical fibers for transcontinental and transoceanic distances. In [24], Sulaiman et al. studied the conformable time-space fractional complex Ginzburg-Landau equation via extended sine-Gordon equation expansion method. In [25], Abdou et al. considered the fractional complex Ginzburg-Landau equation by employing the extended Jacobi elliptic function expansion method. In [26], Arshed constructed the soliton solutions to fractional complex Ginzburg-Landau equation by utilizing the $\exp (-\phi(\xi))$-expansion method. In [27], Ghanbari and Gòmez-Aguilar employed the generalized 
exponential rational function method to study the periodic and hyperbolic soliton solutions to conformable Ginzburg-Landau equation. In [28], Lu et al. studied the $(2+1)$ dimensional fractional complex Ginzburg-Landau equation via the fractional Riccati method and fractional bifunction method. Recently, the complete discrimination system method proposed by Liu is very powerful and useful tool, and the exact solutions of many fractional partial differential equations have been solved (see [29-35]). In this paper, we employed the complete discrimination system method to construct new exact solutions of the fractional complex Ginzburg-Landau equation.

The paper is arranged as follows. In Section 2, we will give the definition of modified Riemann-Liouville derivative and its properties. In Section 3, we will introduce the complete discrimination system for the polynomial method. In Section 4, we will apply this method to solve the fractional complex Ginzburg-Landau equation with the Kerr law and the power law nonlinearity. In Section 5, we draw the numerical simulations. In Section 6, we present the concluding remarks.

\section{Conformable Fractional Derivative and Its Properties}

The definition and properties of the conformable fractional derivative are defined as [36].

Definition 1. Let $f:[0, \infty) \longrightarrow \mathbf{R}$. Then, the conformable fractional derivative of $f$ of order $\alpha$ is defined as

$$
D_{t}^{\alpha} f(t)=\lim _{\varepsilon \longrightarrow 0} \frac{f\left(t+\varepsilon t^{1-\alpha}\right)-f(t)}{\varepsilon}, \quad \forall t \in(0,+\infty), \alpha \in(0,1],
$$

the function $f$ is $\alpha$-conformable differentiable at a point $t$ if the limit in equation (3) exists.

Remark 1. The conformable fractional derivative possesses the following properties:

$$
\begin{aligned}
& \text { (i) } D_{t}^{\alpha}\left(t^{\mu}\right)=\mu t^{\mu-\alpha}, \forall \mu \in \mathbf{R} \\
& \text { (ii) } D_{t}^{\alpha}(a f(t)+b g(t))=a D_{t}^{\alpha} f(t)+b D_{t}^{\alpha} g(t), \\
& \forall a, b \in \mathbf{R} \\
& \text { (iii) } D_{t}^{\alpha}(f \circ g)(t)=\left.t^{1-\alpha} g(t)^{\alpha-1} g^{\prime}(t) D_{t}^{\alpha}(f(t))\right|_{t=g(t)}
\end{aligned}
$$

\section{Complete Discrimination System for the Polynomial}

To show the basic idea of our method, consider the following nonlinear fractional differential equation:

$$
P\left(u, D_{t}^{\alpha} u, D_{x}^{\beta} u, D_{t}^{\alpha} D_{t}^{\alpha} u, D_{t}^{\alpha} D_{x}^{\beta} u, D_{x}^{\beta} D_{x}^{\beta} u, \ldots\right)=0, \quad 0<\alpha, \beta \leq 1,
$$

where $u$ is an unknown function and $P$ is a polynomial of $u$ and its partial fractional derivatives.

Using the fractional complex transformation,

$$
\begin{aligned}
u(x, t) & =u(\xi), \\
\xi & =k \frac{x^{\beta}}{\beta}+\omega \frac{t^{\alpha}}{\alpha},
\end{aligned}
$$

where $k$ and $\omega$ are arbitrary constants.

Equation (4) is reduced to the following integer-order ordinary differential equation:

$$
Q\left(u, u^{\prime}, u^{\prime \prime}, \ldots\right)=0 \text {, }
$$

where $Q$ is a polynomial in $u$ and its derivatives and notation ( 1 ) is the derivative with respect to $\xi$.

Equation (6) can be written as

$$
u^{\prime}(\xi)=G\left(u, \theta_{1}, \theta_{2}, \ldots, \theta_{m}\right)
$$

where $\theta_{1}, \theta_{2}, \ldots, \theta_{m}$ are parameters. Then, integrating the above formula once, we have

$$
\pm\left(\xi-\xi_{0}\right)=\int \frac{\mathrm{d} u}{\sqrt{G\left(u, \theta_{1}, \theta_{2}, \ldots, \theta_{m}\right)}},
$$

where $G(u)$ is a polynomial function and $\xi_{0}$ is an integral constant.

In this paper, there are two complete discrimination system that will be used, the second-order complete discrimination system,

$$
\begin{aligned}
G(u) & =a u^{2}+b u+c, \\
\Delta & =b^{2}-4 a c,
\end{aligned}
$$

and the triple order complete discrimination system for the third degree polynomial,

$$
\begin{aligned}
G(u) & =u^{3}+d_{2} u^{2}+d_{1} u+d_{0}, \\
\Delta & =-27\left(\frac{2 d_{2}^{3}}{27}+d_{0}-\frac{d_{1} d_{2}}{3}\right)^{2}-4\left(d_{1}-\frac{d_{2}^{3}}{3}\right)^{3}, \\
D_{1} & =d_{1}-\frac{d_{2}^{2}}{3} .
\end{aligned}
$$

According to the complete discrimination system for $G(u)$, the roots of $G(u)$ can be classified, and the detailed classification will be given in Section 4 .

\section{Applications}

Taking the fractional complex transformation,

$$
\begin{aligned}
u(x, t) & =U(\xi) e^{i \tau}, \\
\xi & =\frac{x^{2 \delta}}{2 \delta}-\frac{v t^{\delta}}{\delta}, \\
\tau & =-k \frac{x^{2 \delta}}{2 \delta}+\omega \frac{t^{\delta}}{\delta}+\theta,
\end{aligned}
$$

where $v$ is the soliton velocity, $k$ is the soliton frequency, $\omega$ is the soliton wave number, and $\theta$ is the phase constant. 
Inserting (11) into (1) and separating into real and imaginary parts yield

$-\omega U+a\left(U^{\prime \prime}-k^{2} U\right)+b F\left(U^{2}\right) U=2(\alpha-2 \beta) \frac{\left(U^{\prime}\right)^{2}}{U}+2 \alpha U^{\prime \prime}+\gamma U$,

$$
v=-2 a k
$$

Equation (13) gives the velocity of soliton. Taking $\alpha=2 \beta$, equation (12) takes the following form:

$$
(\alpha-4 \beta) U^{\prime \prime}-\left(\omega+a k^{2}+\gamma\right) U+b F\left(U^{2}\right) U=0 .
$$

4.1. Kerr Law. The Kerr law of nonlinearity describes the phenomenon that a light wave in an optical fibre encounters nonlinear responses from nonharmonic motion of electrons with an external electric field. In this case, $F(U)=U$ so that equation (14) reduces to

$$
(\alpha-4 \beta) U^{\prime \prime}-\left(\omega+a k^{2}+\gamma\right) U+b U^{3}=0 .
$$

Multiplying $U^{\prime}$ on both sides of equation (15) and again integrating it on $\xi$, we can obtain

$$
\left(U^{\prime}\right)^{2}=a_{4} U^{4}+a_{2} U^{2}+a_{0}
$$

where $a_{4}=-b / 2(\alpha-4 \beta), a_{2}=\left(\omega+a k^{2}+\gamma\right) /(\alpha-4 \beta)$, and $a_{0}$ are arbitrary constants.

Taking the transformation $U= \pm \sqrt{\left(-2 b /(\alpha-4 \beta)^{-1 / 3} \psi\right.}$ and $\xi_{1}=\left(-2 b /(\alpha-4 \beta)^{1 / 3} \xi\right.$, equation (16) becomes

$$
\left(\psi^{\prime}\right)^{2}=\psi\left(\psi^{2}+p_{1} \psi+p_{0}\right)
$$

where $p_{1}=\left(4\left(\omega+a k^{2}+\gamma\right) /(\alpha-4 \beta)\right)\left(-2 b /(\alpha-4 \beta)^{-2 / 3}\right.$ and $p_{0}=4 a_{0}\left(-2 b /(\alpha-4 \beta)^{-2 / 3}\right.$. Integrating equation (17), we have

$$
\pm\left(\xi_{1}-\xi_{0}\right)=\int \frac{\mathrm{d} \psi}{\sqrt{\psi\left(\psi^{2}+p_{1} \psi+p_{0}\right)}}
$$

where $\xi_{0}$ is the integration constant and values zero in the following solutions. Let $\Delta=p_{1}^{2}-4 p_{0}$ be discriminant of second-order polynomial $G(\psi)=\psi^{2}+p_{1} \psi+p_{0}$, and there are four cases for the solutions of equation (18) according to the cases of roots of $G(\psi)$.

Case $1.1(\Delta=0)$ : as for $\psi>0$, we have

$$
\pm\left(\xi_{1}-\xi_{0}\right)=\int \frac{\mathrm{d} \psi}{\sqrt{\psi}\left(\psi+\left(p_{1} / 2\right)\right)}
$$

If $p_{1}<0$, it follows from equation (19) that the solution of equation (15) takes the form

$$
\begin{aligned}
& u_{1}(x, t)= \pm \sqrt{\frac{\omega+a k^{2}+\gamma}{b^{3}}} \tanh \left\{\left[\frac{\omega+a k^{2}+\gamma}{32 b^{2}(\alpha-4 \beta)}\right]^{1 / 6}\left[\left(\frac{-2 b}{\alpha-4 \beta}\right)^{1 / 3} \xi-\xi_{0}\right]\right\} e^{i\left(\left(-k x^{\delta} / \Gamma(1+\delta)\right)+\left(\omega t^{\delta} / \Gamma(1+\delta)\right)+\theta\right)} \\
& u_{2}(x, t)= \pm \sqrt{\frac{\omega+a k^{2}+\gamma}{b^{3}}} \operatorname{coth}\left\{\left[\frac{\omega+a k^{2}+\gamma}{32 b^{2}(\alpha-4 \beta)}\right]^{1 / 6}\left[\left(\frac{-2 b}{\alpha-4 \beta}\right)^{1 / 3} \xi-\xi_{0}\right]\right\} e^{i\left(\left(-k x^{\delta} / \Gamma(1+\delta)\right)+\left(\omega t^{\delta} / \Gamma(1+\delta)\right)+\theta\right)}
\end{aligned}
$$

If $p_{1}>0$, it follows from equation (19) that the solution of equation (15) takes the form

$$
u_{3}(x, t)= \pm \sqrt{\frac{\omega+a k^{2}+\gamma}{b^{3}}} \tan \left\{\left[\frac{\omega+a k^{2}+\gamma}{32 b^{2}(\alpha-4 \beta)}\right]^{1 / 6}\left[\left(\frac{-2 b}{\alpha-4 \beta}\right)^{1 / 3} \xi-\xi_{0}\right]\right\} e^{i\left(\left(-k x^{\delta} / \Gamma(1+\delta)\right)+\left(\omega t^{\delta} / \Gamma(1+\delta)\right)+\theta\right)}
$$

If $p_{1}=0$, it follows from equation (19) that the solution of equation (15) takes the form

$$
u_{4}(x, t)= \pm \frac{2}{(-2 b /(\alpha-4 \beta))^{1 / 2} \xi-(-2 b /(\alpha-4 \beta))^{1 / 6} \xi_{0}} e^{i\left(\left(-k x^{\delta} / \Gamma(1+\delta)\right)+\left(\omega t^{\delta} / \Gamma(1+\delta)\right)+\theta\right)}
$$

Case $1.2\left(\Delta>0\right.$ and $\left.p_{0}=0\right)$ : as for $\psi>-p_{1}$, we have

$$
\pm\left(\xi_{1}-\xi_{0}\right)=\int \frac{\mathrm{d} \psi}{\psi \sqrt{\psi+p_{1}}}
$$


If $p_{1}>0$, it follows from equation (23) that the solution of equation (15) takes the form

$$
\begin{aligned}
& u_{5}(x, t)= \pm \sqrt{\frac{\omega+a k^{2}+\gamma}{b^{3}}}\left\{\frac{1}{2} \tanh ^{2}\left[\left(\frac{\omega+a k^{2}+\gamma}{32 b^{2}(\alpha-4 \beta)}\right)^{1 / 6}\left(\left(\frac{-2 b}{\alpha-4 \beta}\right)^{1 / 3} \xi-\xi_{0}\right)\right]-1\right\}^{1 / 2} e^{i\left(\left(-k x^{\delta} / \Gamma(1+\delta)\right)+\left(\omega t^{\delta} / \Gamma(1+\delta)\right)+\theta\right)} \\
& u_{6}(x, t)= \pm \sqrt{\frac{\omega+a k^{2}+\gamma}{b^{3}}}\left\{\frac{1}{2} \operatorname{coth}^{2}\left[\left(\frac{\omega+a k^{2}+\gamma}{32 b^{2}(\alpha-4 \beta)}\right)^{1 / 6}\left(\left(\frac{-2 b}{\alpha-4 \beta}\right)^{1 / 3} \xi-\xi_{0}\right)\right]-1\right\}^{1 / 2} e^{i\left(\left(-k x^{\delta} / \Gamma(1+\delta)\right)+\left(\omega t^{\delta} / \Gamma(1+\delta)\right)+\theta\right)}
\end{aligned}
$$

If $p_{1}<0$, it follows from equation (23) that the solution of equation (15) takes the form

$$
u_{7}(x, t)= \pm \sqrt{\frac{\omega+a k^{2}+\gamma}{b^{3}}}\left\{\frac{1}{2} \tan ^{2}\left[\left(\frac{\omega+a k^{2}+\gamma}{32 b^{2}(\alpha-4 \beta)}\right)^{1 / 6}\left(\left(\frac{-2 b}{\alpha-4 \beta}\right)^{1 / 3} \xi-\xi_{0}\right)\right]-1\right\}^{1 / 2} e^{i\left(\left(-k x^{\delta} / \Gamma(1+\delta)\right)+\left(\omega t^{\delta} / \Gamma(1+\delta)\right)+\theta\right)} \text {. }
$$

Case $1.3\left(\Delta>0, p_{0} \neq 0\right)$ : suppose that $\lambda_{1}<\lambda_{2}<\lambda_{3}$, one of $\lambda_{1}, \lambda_{2}, \lambda_{3}$ is zero, and others are two roots of $G(\psi)=0$. Taking the transformation, $\psi=\lambda_{1}+\left(\lambda_{2}-\lambda_{1}\right) \sin ^{2} \varphi$, it is clear that

$$
\pm\left(\xi_{1}-\xi_{0}\right)=\frac{2}{\sqrt{\lambda_{3}-\lambda_{1}}} \int \frac{\mathrm{d} \psi}{\sqrt{1-m_{1}^{2} \sin ^{2} \varphi}},
$$

$$
u_{8}(x, t)= \pm\left(\frac{-2 b}{\alpha-4 \beta}\right)^{1 / 6}\left\{\lambda_{1}+\left(\lambda_{2}-\lambda_{1}\right) s n^{2}\left[\frac{\sqrt{\lambda_{3}-\lambda_{1}}}{2}\left(\left(\frac{-2 b}{\alpha-4 \beta}\right)^{1 / 3} \xi-\xi_{0}\right), m_{1}\right]\right\}^{1 / 2} e^{i\left(\left(-k x^{\delta} / \Gamma(1+\delta)\right)+\left(\omega t^{\delta} / \Gamma(1+\delta)\right)+\theta\right)}
$$

For another transformation, $\psi=\left(-\lambda_{2} \sin ^{2} \varphi+\lambda_{3}\right) /$ $\cos ^{2} \varphi$, it follows from equation (26) that the solution of equation (15) takes the form

$$
\begin{aligned}
u_{9} x, t= \pm & \frac{-2 b}{\alpha-4 \beta} \\
& \cdot\left\{\frac{-\lambda_{2} s n^{2} \sqrt{\lambda_{3}-\lambda_{1}}-2 b / \alpha-4 \beta^{1 / 3} \xi-\xi_{0} / 2, m_{1}+\lambda_{3}}{c n^{2} \sqrt{\lambda_{3}-\lambda_{1}}-2 b / \alpha-4 \beta^{1 / 3} \xi-\xi_{0} / 2, m_{1}}\right\}^{1 / 2} \\
& \cdot e^{i-k x^{\delta} / \Gamma 1+\delta+\omega t^{\delta} / \Gamma 1+\delta+\theta} .
\end{aligned}
$$

where $m_{1}^{2}=\left(\lambda_{2}-\lambda_{1}\right) /\left(\lambda_{3}-\lambda_{1}\right)$.

It follows from equation (26) that the solution of equation (15) takes the form
Case $1.4 \quad(\Delta<0)$ : taking the transformation $\psi=\sqrt{P_{0}} \tan ^{2}(\varphi / 2)$, it is clear that

$$
\pm\left(\xi_{1}-\xi_{0}\right)=p_{0}^{-1 / 4} \int \frac{\mathrm{d} \psi}{\sqrt{1-m_{2}^{2} \sin ^{2} \eta}}
$$

where $m_{2}^{2}=(1 / 2)\left(1-\left(p_{1} / 2 \sqrt{p_{0}}\right)\right)$. It follows from equation (29) that the solution of equation (15) takes the form 


$$
u_{10}(x, t)= \pm\left(\frac{2(\alpha-4 \beta) a_{0}}{-b}\right)^{1 / 4}\left\{\frac{2}{1+c n\left[32 a_{0}^{3}(-2 b /(\alpha-4 \beta))\left((\alpha-4 \beta)^{1 / 3} \xi-\xi_{0}\right), m_{2}\right]}-1\right\}^{1 / 2} e^{i\left(\left(-k x^{\delta} / \Gamma(1+\delta)\right)+\left(\omega t^{\delta} / \Gamma(1+\delta)\right)+\theta\right)}
$$

4.2. Power Law. Power-law nonlinearity can be regarded as a generalisation of Kerrs power-law nonlinearity. In this case, $F(U)=U^{n}$ so that equation (14) can be given as

$$
(\alpha-4 \beta) U^{\prime \prime}-\left(\omega+a k^{2}+\gamma\right) U+b U^{2 n+1}=0,
$$

here, in equation (31) the parameter $n$ dictates the power law nonlinearity. For stability issues, it is necessary to have $0<n<2$, and in particular $n \neq 2$, to avoid self-focusing singularity. Balancing $U^{\prime \prime}$ with $U^{2 n+1}$ in equation (31) gives $N=1 / n$. In order to obtain closed-form solutions, we use the transformation $U=\phi^{1 / 2 n}$ that reduces equation (31) into the ODE:

$$
\begin{aligned}
& (\alpha-4 \beta)\left((1-2 n) \phi^{\prime 2}+2 n \phi \phi^{\prime \prime}\right)-4 n^{2}\left(\omega+a k^{2}+\gamma\right) \phi^{2} \\
& \quad+4 b n^{2} \phi^{3}=0 .
\end{aligned}
$$

Using the balance principle in equation (32),

$$
\left(\phi^{\prime}\right)^{2}=d_{3} \phi^{3}+d_{2} \phi^{2},
$$

where $d_{3}=-4 b n^{2} /((\alpha-4 \beta)(1+n))$ and $d_{2}=4 n^{2}\left(\omega+a k^{2}+\right.$ $\gamma) /(\alpha-4 \beta)$.

Taking the transformation $\phi=\left(-4 b n^{2} /((\alpha-4 \beta)\right.$ $(1+n)))^{1 / 3} \psi$, equation (33) becomes

$$
\left(\psi^{\prime}\right)^{2}=\psi^{3}+d_{2}\left(d_{3}\right)^{-2 / 3} \psi^{2} .
$$

Integrating equation (17), we have

$$
\pm\left(\frac{-4 b n^{2}}{(\alpha-4 \beta)(1+n)}\right)^{1 / 3}\left(\xi-\xi_{0}\right)=\int \frac{\mathrm{d} \psi}{\psi \sqrt{\psi+d_{2}\left(d_{3}\right)^{-2 / 3}}}
$$

We use the complete discrimination system for the third-order polynomial, and then we have the following solving process:

Case 2.1: when $d_{2}>0$, according to Equation (35), we have

$$
\pm\left(\xi-\xi_{0}\right)=\frac{1}{\sqrt{d_{2}\left(d_{3}\right)^{-2 / 3}}} \ln \left|\frac{\sqrt{\psi+d_{2}\left(d_{3}\right)^{-2 / 3}}-\sqrt{d_{2}\left(d_{3}\right)^{-2 / 3}}}{\sqrt{\psi+d_{2}\left(d_{3}\right)^{-2 / 3}}+\sqrt{d_{2}\left(d_{3}\right)^{-2 / 3}}}\right|
$$

then, the solutions of equation (31) can be presented as

$$
\begin{aligned}
u_{11}(x, t)= & \left(\frac{-4 b n^{2}}{(\alpha-4 \beta)(1+n)}\right)^{-1 / 6 n}\left\{\frac{4 n^{2}\left(\omega+a k^{2}+\gamma\right)}{\alpha-4 \beta}\left(\frac{-4 b n^{2}}{(\alpha-4 \beta)(1+n)}\right)^{-2 / 3}\right. \\
& \tanh ^{2}\left[\frac{\left.\left.\sqrt{\left(4 n^{2}\left(\omega+a k^{2}+\gamma\right) /(\alpha-4 \beta)\right)\left(-4 b n^{2} /((\alpha-4 \beta)(1+n))\right)^{-2 / 3}}\left(\frac{-4 b n^{2}}{2}\right)^{-1 / 3}\left(\xi-\xi_{0}\right)\right]\right\}}{(\alpha-4 \beta)(1+n)}\right\}^{1 / 2 n} \\
& \cdot e^{i\left(\left(-k x^{\delta} / \Gamma(1+\delta)\right)+\left(\omega t^{\delta} / \Gamma(1+\delta)\right)+\theta\right)}, \\
u_{12}(x, t)= & \left(\frac{-4 b n^{2}}{(\alpha-4 \beta)(1+n)}\right)^{-1 / 6 n}\left\{\frac{4 n^{2}\left(\omega+a k^{2}+\gamma\right)}{\alpha-4 \beta}\left(\frac{-4 b n^{2}}{(\alpha-4 \beta)(1+n)}\right)^{-2 / 3}\right. \\
& \left.\operatorname{coth}^{2}\left[\frac{\sqrt{\left(4 n^{2}\left(\omega+a k^{2}+\gamma\right) /(\alpha-4 \beta)\right)\left(-4 b n^{2} /((\alpha-4 \beta)(1+n))\right)^{-2 / 3}}}{2}\left(\frac{-4 b n^{2}}{(\alpha-4 \beta)(1+n)}\right)^{-1 / 3}\left(\xi-\xi_{0}\right)\right]\right\} \\
& \cdot e^{i\left(\left(-k x^{\delta} / \Gamma(1+\delta)\right)+\left(\omega t^{\delta} / \Gamma(1+\delta)\right)+\theta\right)} .
\end{aligned}
$$




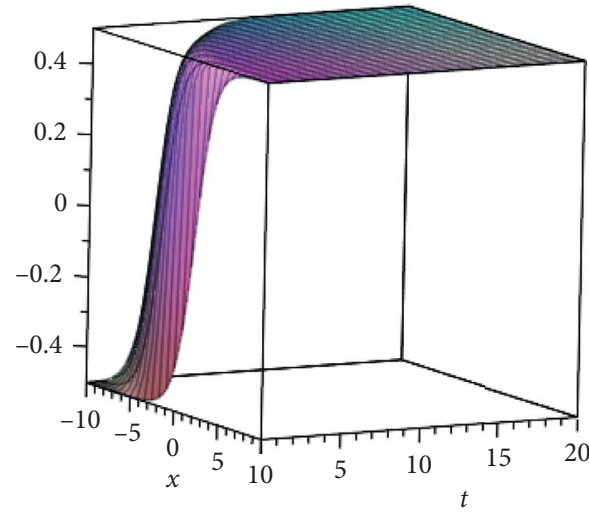

(a)

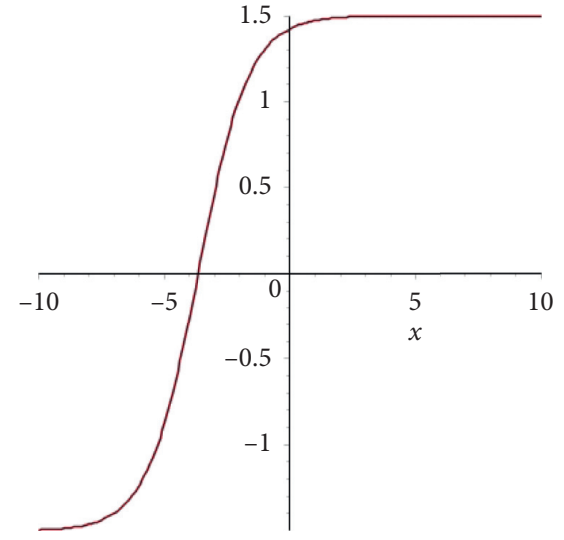

(b)

Figure 1: The solitary wave solution $u_{1}$ for equation (1) with $\omega=1, \alpha=-1, k=1, \gamma=-1, \beta=1 / 4, a=1$, and $b=1$. (a) Perspective view of the wave. (b) The wave along the $z$-axis.

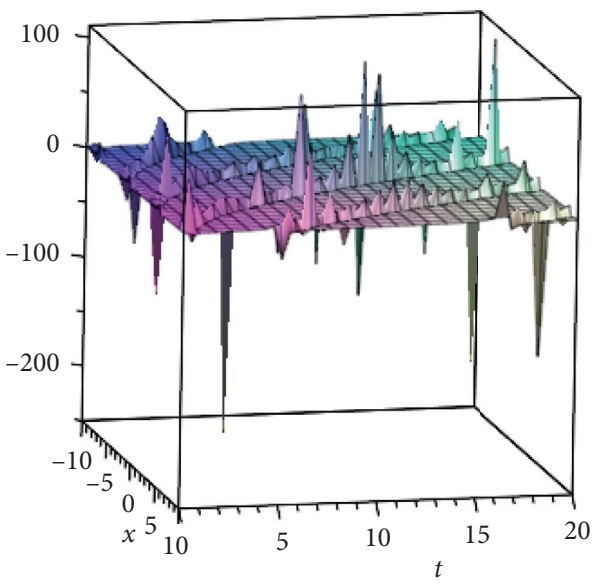

(a)

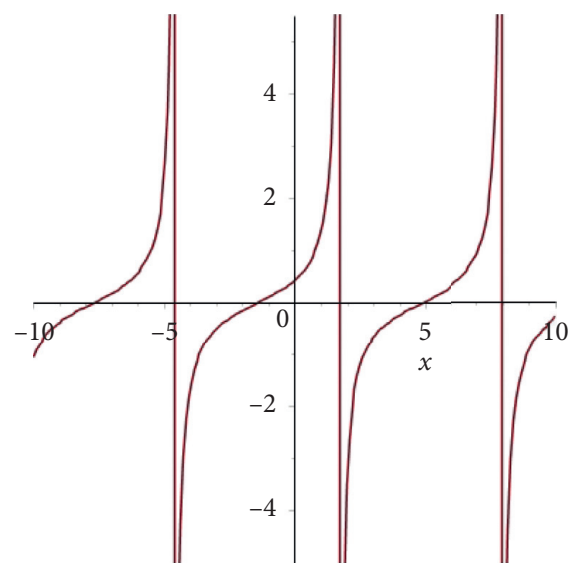

(b)

Figure 2: The triangle function solution $u_{3}$ for equation (1) $\omega=1, \alpha=2, k=1, \gamma=-1, \beta=1 / 4, a=1$, and $b=1$, (a) Perspective view of the wave. (b) The wave along the $z$-axis.

Case 2.2: when $d_{2}<0$, according to equation (35), we have

$$
\pm\left(\xi-\xi_{0}\right)=\frac{2}{\sqrt{-d_{2}\left(d_{3}\right)^{-2 / 3}}} \arctan \sqrt{\frac{\psi+d_{2}\left(d_{3}\right)^{-2 / 3}}{-d_{2}\left(d_{3}\right)^{-2 / 3}}}
$$

$$
\begin{aligned}
u_{13}(x, t)= & \left(\frac{-4 b n^{2}}{(\alpha-4 \beta)(1+n)}\right)^{-1 / 6 n}\left\{-\frac{4 n^{2}\left(\omega+a k^{2}+\gamma\right)}{\alpha-4 \beta}\left(\frac{-4 b n^{2}}{(\alpha-4 \beta)(1+n)}\right)^{-2 / 3}\right. \\
& \left.\left.\tan ^{2}\left[\frac{\sqrt{\left(\left(4 n^{2}\left(\omega+a k^{2}+\gamma\right)\right) /(\alpha-4 \beta)\right)\left(-4 b n^{2} /((\alpha-4 \beta)(1+n))\right)^{-2 / 3}}}{2}\left(\frac{-4 b n^{2}}{(\alpha-4 \beta)(1+n)}\right)^{-1 / 3}\left(\xi-\xi_{0}\right)\right]\right\}\right\}^{1 / 2 n} \\
& \cdot e^{i\left(\left(-k x^{\delta} / \Gamma(1+\delta)\right)+\left(\omega t^{\delta} / \Gamma(1+\delta)\right)+\theta\right)}
\end{aligned}
$$

then, the solutions of equation (31) can be presented as 
Case 2.3: when $d_{2}=0$, according to equation (35), we have

$$
\begin{aligned}
u_{14}(x, t)= & {\left[2\left(\frac{-4 b n^{2}}{(\alpha-4 \beta)(1+n)}\right)^{-1 / 3}\left(\xi-\xi_{0}\right)\right]^{-1 / n} } \\
& \cdot e^{i\left(\left(-k x^{\delta} / \Gamma(1+\delta)\right)+\left(\omega t^{\delta} / \Gamma(1+\delta)\right)+\theta\right)} .
\end{aligned}
$$

\section{Graphical Representation of the Obtained Solutions}

In this section, the exact solutions of the fractional complex Ginzburg-Landau equation are given. Through the above results, we get some new exact solutions, such as solitary wave solutions $u_{1}(x, t), u_{2}(x, t), u_{5}(x, t), u_{6}(x, t), u_{11}(x, t)$, and $u_{12}(x, t)$; trigonometric function solutions $u_{3}(x, t)$, $u_{7}(x, t)$, and $u_{13}(x, t)$; Jacobi elliptic function double periodic solutions $u_{8}(x, t), u_{9}(x, t)$, and $u_{10}(x, t)$; rational function solutions $u_{4}(x, t)$ and $u_{14}(x, t)$. Furthermore, $u_{1}(x, t)$ and $u_{5}(x, t)$ are bounded solutions and $u_{2}(x, t)$ and $u_{6}(x, t)$ are unbounded solutions. Comparing with other works $[25,26]$, these new solutions have not been reported in the former literature. Using the mathematical software Maple, we plot some of these obtained solutions which are shown in Figures 1 and 2.

\section{Conclusion}

In this work, we apply the complete discrimination system method to construct the exact solution to fractional complex Ginzburg-Landau equation with Kerr and power laws of nonlinearity. The classification of all traveling wave solutions are given by the complete discrimination system, and these exact solutions include solitary wave solutions, rational function solutions, Jacobian elliptic function solutions, and triangle function solutions. Comparing with other works $[25,26]$, these solutions have not been reported in the former literature. Moreover, this method is very efficient and powerful in finding the exact solutions for the nonlinear fractional differential equations, and the obtained solutions can help us to more deeply explain the nonlinear dynamics of optical soliton propagations.

\section{Data Availability}

No data were used to support this study.

\section{Conflicts of Interest}

The author declares that there are no conflicts of interest regarding the publication of this paper.

\section{Authors' Contributions}

All authors read and approved the final manuscript.

\section{Acknowledgments}

This work was supported by Science Research Fund of Education Department of Sichuan Province of China under Grant no. 18ZB0537.

\section{References}

[1] B. Ghanabari and D. Baleanu, "A novel technique to construct exact solutions for nonlinear partial differential equations," The European Physical Journal Plus, vol. 134, Article ID 506, 2019.

[2] Y.-M. Chu, M. A. Shallal, S. Mehdi Mirhosseini-Alizamini, H. Rezazadeh, S. Javeed, and D. Baleanu, "Application of modified extended Tanh technique for solving complex Ginzburg-Landau equation considering Kerr law nonlinearity," Computers, Materials \& Continua, vol. 66, no. 2, pp. 1369-1378, 2020.

[3] N. H. Tuan, T. B. Ngoc, D. Baleanu, and D. O'Regan, “On well-posedness of the sub-diffusion equation with conformable derivative model," Communications in Nonlinear Science and Numerical Simulation, vol. 89, Article ID 105332, 2020.

[4] W. J. Zhu, Y. H. Xia, and Y. Z. Bai, “Traveling wave solutions of the complex Ginzburg-Landau equation with Kerr law nonlinearity," Applied Mathematics and Computation, vol. 382, Article ID 125342, 2020.

[5] M. S. Osman, B. Ghanbari, and J. A. T. Machado, "New complex waves in nonlinear optics based on the complex Ginzburg-Landau equation with Kerr law nonlinearity," European Physical Journal Plus, vol. 134, no. 1, pp. 20-30, 2019.

[6] C. S. Liu, "Exact traveling wave solutions for a kind of generalized ginzburg-landau equation," Communications in Theoretical Physics, vol. 43, pp. 787-790, 2005.

[7] A. Biswas and R. T. Alqahtani, "Optical soliton perturbation with complex Ginzburg-Landau equation by semi-inverse variational principle," Optik, vol. 147, pp. 77-81, 2017.

[8] W. Gao, H. M. Baskonus, and L. Shi, "New investigation of bats-hosts-reservoir-people coronavirus model and application to 2019-nCoV system," Advance in Difference Equations, vol. 2020, Article ID 391, 2020.

[9] H. M. Baskonus, "Complex surfaces to the fractional $(2+1)$ dimensional boussinesq dynamical model with the local M-derivative," The European Physical Journal Plus, vol. 134, Article ID 322, 2019.

[10] E. İlhan and İ. O. Kiymaz, "A generalization of truncated M-fractional derivative and applications to fractional differential equations," Applied Mathematics and Nonlinear Sciences, vol. 5, no. 1, pp. 171-188, 2020.

[11] N. Valliammal, C. Ravichandran, Z. Hammouch, and H. M. Baskonus, "A new investigation on fractional-ordered neutral differential systems with state-dependent delay," International Journal of Nonlinear Sciences and Numerical Simulation, vol. 20, no. 7-8, p. 803C809, 2019.

[12] Y. Zhang, C. Cattani, and X. J. Yang, "Local fractional homotopy perturbation method for solving non-homogeneous heat conduction equations in fractal domains," Entropy, vol. 17, no. 10, pp. 6753-6764, 2015.

[13] W. Gao, G. Yel, H. Mehmet Baskonus, and C. Cattani, "Complex solitons in the conformable $(2+1)$-dimensional ablowitz-kaup-newell-segur equation," Aims Mathematics, vol. 5 , no. 1, p. 507, 2020.

[14] A. Yokuş and S. Gülbahar, "Numerical solutions with linearization techniques of the fractional Harry Dym equation," 
Applied Mathematics and Nonlinear Sciences, vol. 4, no. 1, pp. 35-42, 2019.

[15] J. Danane, K. Allali, and Z. Hammouch, "Mathematical analysis of a fractional differential model of HBV infection with antibody immune response," Chaos, Solitons \& Fractals, vol. 136, Article ID 109787, 2020.

[16] S. Arshed, A. Biswas, and F. Mallawi, "Optical solitons with complex Ginzburg-Landau equation having three nonlinear forms," Physics Letters A, vol. 383, no. 36, Article ID 126026, 2019.

[17] A. Biswas, Y. Yildirim, E. Yasar et al., "Optical soliton perturbation with complex Ginzburg-Landau equation using trial solution approach," Optik, vol. 160, pp. 44-60, 2018.

[18] A. Das, A. Biswas, M. Ekici, Q. Zhou, A. S. Alshomrani, and M. R. Belic, "Optical solitons with complex Ginzburg-Landau equation for two nonlinear forms using F-expansion," Chinese Journal of Physics, vol. 61, pp. 255-261, 2019.

[19] M. Inc, A. I. Aliyu, A. Yusuf, and D. Baleanu, "Optical solitons for complex Ginzburg-Landau model in nonlinear optics," Optik, vol. 158, pp. 368-375, 2018.

[20] H. Rezazadeh, "New solitons solutions of the complex Ginzburg-Landau equation with Kerr law nonlinearity," Optik, vol. 167, pp. 218-227, 2018.

[21] J. Li and J. Shi, "Bifurcations and exact solutions of ac-driven complex Ginzburg-Landau equation," Applied Mathematics and Computation, vol. 221, pp. 102-110, 2013.

[22] Z. Li and T. Y. Han, "Bifurcation and exact solutions for the $(2+1)$-dimensional conformable time-fractional Zoomeron equation," Advance in Difference Equations, vol. 2020, Article ID 656, 2020.

[23] Z. Li, T. Y. Han, and C. Huang, "Bifurcation and new exact traveling wave solutions for time-space fractional Phi-4 equation," AIP Advance, vol. 10, Article ID 115113, 2020.

[24] T. A. Sulaiman, H. M. Baskonus, and A. Bulut, "Optical solitons and other solutions to the conformable space-time fractional complex Ginzburg-Landau equation under Kerr law nonlinearity," Pramana: Journal of Physics, vol. 91, p. 4, 2018.

[25] M. A. Abdou, A. A. Soliman, A. Biswas, M. Ekici, Q. Zhou, and S. P. Moshokoa, "Dark-singular combo optical solitons with fractional complex Ginzburg-Landau equation," Optik, vol. 171, pp. 463-467, 2018.

[26] S. Arshed, "Soliton solutions of fractional complex GinzburgLandau equation with Kerr law and non-Kerr law media," Optik, vol. 160, pp. 322-332, 2018.

[27] B. Ghanbari and J. F. Gò.an-Aguilar, "Optical soliton solutions of the Ginzburg-Landau equation with conformable derivative and Kerr law nonlinearity," Revista Mexicana de Fisica, vol. 65, pp. 73-81, 2019.

[28] P. H. Lu, B. H. Wang, and C. Q. Dai, "Fractional traveling wave solutions of the $(2+1)$ - dimensional fractional complex Ginzburg-Landau equation via two methods," Mathematical Methods in the Applied Sciences, vol. 43, pp. 1-9, 2020.

[29] C.-s. Liu, "Applications of complete discrimination system for polynomial for classifications of traveling wave solutions to nonlinear differential equations," Computer Physics Communications, vol. 181, no. 2, pp. 317-324, 2010.

[30] C. S. Liu, "Classification of all single travelling wave solutions to Calogero-Degasperis-Focas equation," Communications in Theoretical Physics, vol. 48, no. 4, pp. 601-604, 2007.

[31] D. Cao, C. Li, and J. He, "Exact solutions to the space-time fraction Whitham-Broer-Kaup equation," Modern Physics Letters B, vol. 34, p. 16, 2020.
[32] Y. Kai, B. L. Zheng, and W. Y. Xu, "Exact single traveling wave solutions to generalized $(2+1)$-dimensional Gardner equation with variable coefficients," Results in Physics, vol. 15, 2019.

[33] B. Guan, S. B. Li, S. Q. Chen, L. G. Zhang, and C. H. Wang, "The classification of single traveling wave solutions to coupled time-fractional KdV-Drinfel'd-Sokolov-Wilson system," Results in Physics, vol. 13, Article ID 102291, 2019.

[34] Y. Liu and X. Wang, "The construction of solutions to Zakharov-Kuznetsov equation with fractional power nonlinear terms," Advances in Difference Equations, vol. 2019, Article ID 134, 2019.

[35] Z. Li, T. Y. Han, and C. Huang, "Exact single traveling wave solutions for generalized fractional gardner equations," Mathematical Problems in Engineering, vol. 2020, Article ID 8842496, 10 pages, 2020.

[36] R. Khalil, M. Al Horani, A. Yousef, and M. Sababheh, "A new definition of fractional derivative," Journal of Computational and Applied Mathematics, vol. 264, pp. 65-70, 2014. 